\title{
Factores de riesgo y síndrome del túnel carpiano en el entorno laboral
}

\section{Risk Factors and Carpal Tunnel Syndrome in the Work Environment}

\author{
Ángel Sutil Blanco ${ }^{1}$ Silvia Bernaldo de Quirós Ramos ${ }^{1}$ David Varillas Delgado 2,3 \\ Fernando García de Lucas ${ }^{1}$ \\ ${ }^{1}$ Servicio de Cirugía Ortopédica y Traumatología, Hospital FREMAP, \\ Address for correspondence Ángel Sutil Blanco, Servicio de Cirugía \\ Majadahonda, Madrid, España \\ ${ }^{2}$ Servicios Auxiliares, VEMESA, Madrid, España \\ ${ }^{3}$ Facultad de Ciencias de la Salud, Unidad de Apoyo a la Investigación, \\ Ortopédica y Traumatología, Hospital FREMAP, Majadahonda, Madrid, \\ España (e-mail: angel_sutil@fremap.es).
} Universidad Francisco de Vitoria, Pozuelo de Alarcón, Madrid, España

Rev Iberam Cir Mano 2018;46:20-25.

\begin{abstract}
Resumen
Introducción Las neuropatías compresivas son las patologías neurológicas periféricas más frecuentes del miembro superior, suponiendo una de las principales causas de pérdida de días laborales. El síndrome del túnel del carpo (STC), es la más frecuente, y se produce por un atrapamiento del nervio mediano a su paso por el túnel carpiano resultando en un daño de tipo isquémico y mecánico. El objetivo de este estudio, fue investigar la relación causal entre la exposición a distintos factores de riesgo laborales y el STC en nuestro medio.

Material y Método Estudio descriptivo, observacional de casos-control restrospectivo. Doscientos veinte pacientes fueron diagnosticados con STC en el área de influencia de nuestro centro hospitalario. Se efectuó tratamiento quirúrgico en pacientes con manifestaciones clínico-electroneurofisiológicas de intensidad moderada-severa y falta de respuesta a medidas conservadoras.

Resultados Noventa y un pacientes fueron hombres $(41,4 \%$ ) y 129 mujeres $(58,6 \%)$, todos en edad laboral, con una edad media de 45,26 años. El 85\% presentaban alta repetición de movimientos de la muñeca. La mayoría presentaba afectada la extremidad dominante. Fueron intervenidos mediante descompresión quirúrgica 191 pacientes y 29 se trataron con medidas conservadoras. Se estudió la prevalencia del STC según los distintos gremios de trabajadores y factores de riesgo. Conclusión Existe un mayor riesgo en profesiones con elevada repetición de movimientos

Palabras Clave

- neuropatías compresivas

- lesiones de muñeca

- síndrome del túnel del carpo de muñeca, de padecer STC con respecto a las profesiones que tienen baja repetición. La afectación de la mano no dominante tiene un riesgo menor que la mano dominante o la bilateral. El sexo femenino, obesidad, diabetes, artritis reumatoide e hiperuricemia, presentan mayor riesgo de STC, aunque no estadísticamente significativo. Como conclusión, existen determinadas profesiones que pueden aumentar el riesgo de padecer este síndrome, así como patologías endocrinas. Sin embargo, no se puede asumir que exista una relación causal suficiente para desencadenarlo.
\end{abstract}

received

November 10, 2017

accepted

March 26, 2018

published online

May 2, 2018
DOI https://doi.org/

10.1055/s-0038-1642596. ISSN $1698-8396$.
Copyright $\odot 2018$ Thieme Revinter

Publicações Ltda, Rio de Janeiro, Brazil
License terms

(ㄷ) (i) $\ominus$ (\$) 
Abstract

\section{Keywords}

- entrapment neuropathies

- wrist injuries

- carpal tunnel syndrome
Introduction Compressive neuropathies are the most frequent peripheral neurological pathologies of the upper limb assuming one of the leading causes of loss of workdays. Carpal tunnel syndrome (CTS) is the most common, caused by an entrapment of the median nerve as it passes through the carpal tunnel, resulting in ischemic and mechanical damage. The objective was to investigate the causal relationship between exposure to occupational risk factors and carpal tunnel syndrome.

Material and Method retrospective, descriptive, observational case-control study. 220 patients were diagnosed with carpal tunnel syndrome in the area of influence of our hospital center. Surgical treatment was performed in patients with clinicalelectroneurophysiologicals manifestations of moderate-severe intensity and lack of response to conservative measures.

Results 91 patients were men (41.4\%) and 129 women (58.6\%), all working age, with an average age of 45.26 years. $85 \%$ had high repetition of wrist movements. dominant extremity was most presented affected. Were operated by surgical decompression 191 patients and 29 were treated with conservative measures. Carpal tunnel syndrome prevalence was studied according to the different workers ' unions and risk factors.

Conclusion There is a greater risk in professions with high repetition of wrist movements to suffer carpal tunnel syndrome respect to the professions that had low repetition. Non-dominant hand involvement has a lower risk than the dominant or bilateral hand. The female sex, obesity, diabetes, rheumatoid arthritis and hyperuricemia, present greater risk of carpal tunnel syndrome but not statistically significant. In conclusion, there are certain professions that can increase the risk of developing this syndrome, as well as endocrine pathologies. However, it cannot be assumed that there is sufficient causal relationship to trigger it.

\section{Introducción}

Las neuropatías compresivas son las patologías neurológicas periféricas más frecuentes del miembro superior y son una de las causas más importantes de pérdida de días laborales en las empresas. ${ }^{1}$

Dentro de las neuropatías compresivas, el síndrome del túnel del carpo (STC) es la más frecuente, y se produce por un atrapamiento del nervio mediano en el túnel carpiano (formado por el retináculo flexor y los huesos del carpo), aumentando la presión sobre el nervio, lo que da lugar a un daño de tipo isquémico y mecánico sobre el mismo. El resultado es la presencia de dolor y parestesias en territorio del nervio mediano en la mano con empeoramiento nocturno, pudiendo producir pérdida sensorial permanente y déficit motor si esa compresión es muy prolongada en el tiempo. ${ }^{2,3}$

Se ha demostrado que las mujeres tienen mayor riesgo de padecerlo, siendo la distribución por edad bimodal, con un pico entre los 50 y los 59 años y un segundo pico por encima de los 70 años. ${ }^{4}$ Su prevalencia es variable en función de la inclusión de criterios clínicos y/o electroneurofisiológicos en su definición; así se observa una prevalencia de un 3,72\% en la población de los Estados Unidos de América, ${ }^{5}$ y en España de un $3 \%$ según datos facilitados por el Ministerio de Empleo y Seguridad Social de España (2014).

El STC es reconocido como enfermedad profesional según el R.D. $1299 / 2006$, siendo el responsable del $24,1 \%$ de todas las enfermedades profesionales registradas por el Área de Prevención de FREMAP.

Varios autores han intentado establecer una relación del STC con la actividad laboral en la que se realizan esfuerzos y movimientos repetitivos, posturas forzadas mantenidas o se manejan herramientas que producen vibración, ${ }^{6-8}$ de forma que los sujetos sometidos a esos factores de riesgo tienen una mayor incidencia de padecer una compresión del nervio mediano a su paso por el túnel del carpo.

El objetivo de este trabajo fue investigar la relación causal entre la exposición de distintos factores de riesgo laborales y el STC.

\section{Material y Método}

\section{- Diseño del Estudio}

Estudio observacional transversal de casos-control, retrospectivo.

\section{- Pacientes}

Se obtuvieron datos de un total de 220 pacientes diagnosticados de síndrome del túnel del carpo en el área de influencia de nuestro centro hospitalario, durante el periodo comprendido entre el $1^{\circ}$ de enero de 2014 y el 31 de julio de 2015. El estudio fue aprobado por el comité de ética de nuestro hospital. 
Los pacientes seleccionados como casos fueron aquellos que presentaron STCy controles aquellos pacientes que pertenecen al mismo gremio profesional y que no desarrollaron STC.

Se seleccionaron del total, un conjunto de pacientes que cumplían como criterios de inclusión, anamnesis, exploración física y estudio electroneurofisiológico compatibles para poder considerar el diagnóstico:

- Historia clínica: Presencia de disestesias en el territorio de inervación del nervio mediano, así como aparición de parestesias nocturnas.

- Exploración física: Positividad para una o más de las siguientes pruebas de provocación: Tinel, Durkan y "scratch collapse test."

- Estudio electroneurofisiológico: Valoración de los siguientes parámetros:

a) Existencia de disminución de la velocidad de conducción sensitiva inferior $50 \mathrm{~m} / \mathrm{s}$.

b) Latencia motora distal determinado en milisegundos (ms), interpretando resultados como leves si fueron inferiores a 4 milisegundos, moderados inferiores 4-6 milisegundos y severos cuando fueron superiores a 6 milisegundos.

Fueron excluidos del estudio aquellos pacientes que no se encontraban en edad laboral comprendida entre 18 y 65 años, ausencia de diagnóstico clínico-neurofisiológico, negación explícita de participación en el estudio, síndrome del túnel del carpo diagnosticado en el embarazo o en el contexto de procesos patológicos asociados al sistema nervioso periférico como polineuropatías.

\section{- Variables}

Se recogieron las siguientes variables demográficas: sexo, edad, peso, altura, índice de masa corporal (IMC), profesión mostrando elevada o baja repetición de movimientos a la que se somete la articulación radiocarpiana; entendiendo como movimiento repetitivo aquel cuyo ciclo es menor de 30 segundos en el que se repite la misma clase de acción técnica en más del 50\% de la jornada, según la norma UNE EN 1005-5:2007 ("normativa técnica sobre trabajos repetitivos" Instituto nacional de seguridad e higiene en el trabajo Ministerio de trabajo e inmigración, Gobierno de España). Esa normativa recoge una evaluación del riesgo asociado a trabajos que implican alta o baja repetición de movimientos, la cual especifica que existe riesgo cuando el ángulo articular es mayor del 50\% del rango articular máximo el $25 \%$ de la jornada, es decir, mantener dorsiflexión o flexión palmar de muñeca más allá de $45^{\circ}$, desviación radial superior a $15^{\circ}$, desviación cubital superior a $20^{\circ}$.

También se consideró dominancia de mano afectada, así como la presencia de bilateralidad del proceso.

Se determinó la duración de la jornada laboral diaria, el tiempo total de permanencia en dicho trabajo, y la presencia de factores clínicos asociados, tales como diabetes, obesidad $\mathrm{y}$ artritis reumatoide.

La obesidad puede tener influencia a su vez en la gravedad del STC. Se calcularon los pesos de referencia ${ }^{9}$ y la obesidad se definió como un peso que excedía el valor de referencia por encima del $10 \%$. Se recogieron datos sobre enfermedades que predisponían a padecer STC; como diabetes, artritis reumatoide e hiperuricemia.

\section{- Procedimiento}

Se realizó tratamiento quirúrgico en aquellos pacientes que presentaban manifestaciones clínicas de intensidad moderada-severa, tales como: parestesias, adormecimiento constante, disfunción motora, atrofia de la musculatura tenar, falta de respuesta a medidas conservadoras. El resto de pacientes no se sometieron a intervención quirúrgica y fueron tratados de forma conservadora.

\section{Análisis Estadístico}

Se efectuó mediante el programa SPSS $20.0^{\circledR}$, realizando un análisis descriptivo, representando las variables cuantitativas mediante media y desviación estándar y las variables cualitativas por porcentajes y tantos por ciento. Se utilizó regresión logística binaria para calcular los valores p de los odds-ratio y el $95 \%$ de los intervalos de confianza en los factores de riesgo que determinaran STC. El test Hoster Lemesnov se usó para determinar la bondad de ajuste de los valores presentados para la utilización de la regresión. Se efectuó test $\mathrm{T}$ de Student en la comparativa del riesgo de padecer STC en el tiempo de trabajo y duración del mismo.

Los resultados con valores $p<0,05$ se mostraron como estadísticamente significativos.

\section{Resultados}

Los datos de edades se mostraron en la - Tabla 1. Fueron intervenidos quirúrgicamente, mediante descompresión abierta del nervio mediano en el túnel carpiano, 191 pacientes ( $86,8 \%)$, y $29(13,2 \%)$ fueron tratados con medidas conservadoras.

También se diferenciaron dos grupos, aquellos pacientes que presentaban una elevada repetición de movimientos en la muñeca, fueron 187 pacientes (el 85\%), y los que presentaban una baja repetición, 33 pacientes (el 15\%).

Se estudió la prevalencia del STC en la mano dominante, la cual estaba afectada en 151 pacientes $(68,7 \%$ ) y la no dominante en 41 pacientes (18,5\%), siendo bilateral en 28 pacientes (12,8\%).

Valorando el sobrepeso, 131 pacientes (59,6\%) presentaban un IMC $>25$ (sobrepeso u obesidad), mientras que $89(40,4 \%)$ presentaban un IMC $<25$.

Tabla 1 Pacientes afectados de síndrome de túnel del carpo

\begin{tabular}{|l|l|l|}
\hline & Media (SD)* & Rango \\
\hline Edad & 45,3 años $( \pm 8,384$ años $)$ & 44 \\
\hline $\begin{array}{l}\text { Tiempo trabajo } \\
\text { (horas/día) }\end{array}$ & $\begin{array}{l}7,7 \text { horas } \\
( \pm 0,938 \text { horas })\end{array}$ & 6 \\
\hline $\begin{array}{l}\text { Duración trabajo } \\
\text { (meses) }\end{array}$ & $\begin{array}{l}96,55 \text { meses } \\
( \pm 87,55 \text { meses })\end{array}$ & 469 \\
\hline
\end{tabular}

(SD)* Desviación estándar. 
Tabla 2 Frecuencias de los distintos factores de riesgo en pacientes del estudio

\begin{tabular}{|l|l|}
\hline & Frecuencia \\
\hline Diabetes & $8(3,6 \%)$ \\
\hline Artritis reumatoide & $2(0,9 \%)$ \\
\hline Hiperuricemia & $10(4,5 \%)$ \\
\hline
\end{tabular}

Las horas diarias dedicadas a la actividad laboral en los pacientes del estudio, así como la permanencia en ese puesto de trabajo se mostró en la - Tabla 1.

Se valoraron otros factores que pudieran estar relacionados con el desarrollo del STC, como diabetes, artritis reumatoide e hiperuricemia ( - Tabla 2 ).

La prevalencia de STC en los distintos gremios asegurados en la red de hospitales de FREMAP se muestra en la - Tabla 3.

Para el estudio de la regresión logística, primeramente se efectuó test de bondad de ajuste Hoster Lemesnov con valor de $p=0,299$.

Tabla 3 Prevalencia de STC en nuestra red de hospitales

\begin{tabular}{|l|l|l|l|}
\hline & STC & Asegurados & Prevalencia \\
\hline Picador (minería/cantera) & 3 & 2112 & $0,14 \%$ \\
\hline $\begin{array}{l}\text { Técnico } \\
\text { (instalador de aire, } \\
\text { montaje exteriores) }\end{array}$ & 9 & 6060 & $0,15 \%$ \\
\hline Administrativo & 19 & 140543 & $0,01 \%$ \\
\hline Limpieza & 30 & 53072 & $0,06 \%$ \\
\hline $\begin{array}{l}\text { Carnicero/Pescadero/ } \\
\text { Deshuesador/Panadero }\end{array}$ & 32 & 13137 & $0,24 \%$ \\
\hline $\begin{array}{l}\text { Sanitario } \\
\text { (enfermero/ } \\
\text { auxiliar enfermería) }\end{array}$ & 7 & 101127 & $0,01 \%$ \\
\hline Rotulista & 1 & 4950 & $0,02 \%$ \\
\hline Cajera/Ventas/Reponedora & 17 & 103687 & $0,01 \%$ \\
\hline Industria-Fábrica & 28 & 70007 & $0,04 \%$ \\
\hline Peluquería & 9 & 16163 & $0,05 \%$ \\
\hline $\begin{array}{l}\text { Hostelería } \\
\text { (camarero/cocinero) }\end{array}$ & 16 & 86058 & $0,02 \%$ \\
\hline Jardinero & 4 & 4045 & $0,10 \%$ \\
\hline Carpintero/Soldador & 4 & 7048 & $0,06 \%$ \\
\hline $\begin{array}{l}\text { Educación } \\
\text { (profesora/guardería) }\end{array}$ & 2 & 71181 & $0,00 \%$ \\
\hline Costurera & 1 & 849 & $0,12 \%$ \\
\hline Albañil/Obrero/Encofrador & 16 & 68319 & $0,02 \%$ \\
\hline Pintor/Barnizador & 4 & 1440 & $0,28 \%$ \\
\hline Mecánico & 10 & 14390 & $0,07 \%$ \\
\hline Conductor/Maquinista & 5 & 37212 & $0,01 \%$ \\
\hline Agricultor/Ganadero & 2 & 33791 & $0,01 \%$ \\
\hline Farmacéutico & 1 & 4986 & $0,02 \%$ \\
\hline
\end{tabular}

Tabla 4 Riesgo de padecer STC en los pacientes del estudio en los distintos gremios de trabajo

\begin{tabular}{|c|c|c|c|}
\hline & & Odd Ratio 95\% IC & $P$ valor \\
\hline \multirow{2}{*}{$\begin{array}{l}\text { Repetición } \\
\text { muñeca }\end{array}$} & Alta & $1,253(0,409-3,839)$ & \multirow[t]{2}{*}{$p=0,693$} \\
\hline & Baja & 1 & \\
\hline \multirow[t]{3}{*}{ Sexo } & Hombre & 1 & \multirow[t]{3}{*}{$p=0,108$} \\
\hline & Mujer & $1,964(0,863-4,468)$ & \\
\hline & $20-34$ & 1 & \\
\hline \multirow[t]{2}{*}{ Edad } & $35-49$ & $0,933(0,513-1,695)$ & \multirow[t]{2}{*}{$p=0,819$} \\
\hline & $50-64$ & 1 & \\
\hline \multirow[t]{3}{*}{ IMC } & $<25$ & 1 & \\
\hline & $>25$ & $1,078(0,498-2,333)$ & $p=0,850$ \\
\hline & No & $0,774(0,432-1,385)$ & $p=0,774$ \\
\hline \multirow[t]{2}{*}{ Dominancia } & Si & 1 & \\
\hline & Bilateral & 1 & \\
\hline \multirow[t]{2}{*}{ Diabetes } & Si & $1,179(0,140-9,920)$ & \multirow[t]{2}{*}{$p=0,879$} \\
\hline & No & 1 & \\
\hline \multirow{2}{*}{$\begin{array}{l}\text { Artritis } \\
\text { reumatoide }\end{array}$} & Si & $1,324(0,237-3,218)$ & \multirow[t]{2}{*}{$p=0,578$} \\
\hline & No & 1 & \\
\hline \multirow[t]{2}{*}{ Hiperuricemia } & Si & $1,213(0,524-4,314)$ & \multirow[t]{2}{*}{$p=0,783$} \\
\hline & No & 1 & \\
\hline
\end{tabular}

Se estudió la relación entre la actividad laboral y el riesgo de padecer STC, viendo que los sujetos con elevada repetición de movimientos de muñeca tuvieron un odd ratio de sufrir STC de $1,253(p=0,693)$ con respecto a los pacientes con trabajos de baja repetición de movimientos (-Tabla 4); lo mismo con el sexo, observando que las mujeres presentaron un odd ratio de 1,964 ( $p=0,108$ ) de intervención quirúrgica por STC sobre los hombres. Se mostraron también los odd ratios de la edad (odd ratio $0,933(p=0,819)$ ), la obesidad (odd ratio $1,078(p=0,850)$ ) y la dominancia de la mano (odd ratio $0,774(p=0,388)$ ) ( - Tabla 4 ).

En lo referente a las enfermedades que pueden estar en relación con el STC, se estudió la diabetes (odd ratio 1,179 $(p=0,879))$, artritis reumatoide (odd ratio $1,324(p=0,578)$ ), e hiperuricemia (odd ratio $1,213(p=0,783)$ ) ( Tabla 4 ).

En cuanto al tiempo de la jornada laboral en pacientes con STC, se estudió el riesgo de sufrir STC, al igual que los años de permanencia en ese trabajo, con resultados sin significancia estadística $p=0,409$ y $p=0,546$ respectivamente ( - Tabla 5).

Tabla 5 Comparativa del riesgo de padecer STC en relación a la duración de la jornada laboral y el tiempo de permanencia en el trabajo

\begin{tabular}{|l|l|}
\hline & $P$ valor \\
\hline Tiempo de trabajo (h/día) & $p=0,409$ \\
\hline Duración del trabajo (meses) & $p=0,546$ \\
\hline
\end{tabular}




\section{Discusión}

Existe una clara relación descrita en la literatura entre el STC y la actividad laboral, en la cual se realizan esfuerzos y movimientos repetitivos, posturas forzadas mantenidas, o se manejan herramientas que producen impacto o vibración; ${ }^{7,8}$ de tal forma que los sujetos sometidos a esos factores de riesgo tienen una mayor incidencia de padecer una compresión del nervio mediano a su paso por el túnel del carpo.

En el trabajo presentado, todos los individuos presentaron actividad laboral en el momento del diagnóstico. Algunos trabajos, como el presentado por Day DC y col. y Gruson y col., ponen de manifiesto que el comportamiento y el manejo clínico-terapéutico de los pacientes en el entorno laboral, en ocasiones difieren de aquellos pertenecientes al ámbito no laboral. ${ }^{10,11}$ Presentan mayor porcentaje de intervenciones quirúrgicas, resultados clínicos subóptimos, sintomatología tras intervención mantenida o fluctuante sin mejoría subjetiva, necesidad de mayores tiempos de recuperación hasta retomar la actividad y existencia de potenciales beneficios secundarios asociados con la enfermedad. Por tanto, no podemos contrastar la existencia de diferencias con respecto a pacientes no laborales diagnosticados de STC.

Se ha demostrado en la literatura que el uso de herramientas que producen vibración mantenida y el hecho de realizar una fuerza de prensión mayor a $4 \mathrm{~kg}$, suponen factores de riesgo para desarrollar esa patología; ${ }^{12}$ aunque esas variables no han sido consideradas en el presente estudio.

Es importante señalar, que se evalúa la relación de causalidad entre los diferentes tipos de profesiones y el desarrollo del STC, contemplando unos valores de referencia heterogéneos en cuanto a rangos y número de repeticiones de movilidad de muñeca; lo cual dificulta la comparación entre los mismos en el momento de considerar que una profesión objeto de estudio, pueda tener un mayor o menor riesgo de presentar STC.

Los resultados de este estudio mostraron una mayor prevalencia del STC en aquellas profesiones dónde se desarrolla una elevada repetición de movimientos y un amplio rango en la movilidad de la muñeca a lo largo de su jornada laboral (odd ratio 1,253), tomando como referencia la norma UNE EN 1005-5:2007; resultados que concuerdan con otras publicaciones actuales como la de Shiri $\mathrm{R} \mathrm{y} \mathrm{col.}{ }^{13}$

Coincidiendo con otros trabajos, se encontró una mayor incidencia de STC en mujeres que en hombres, mostrando un mayor riesgo de padecerlo, (odd ratio 1,964) y de recibir tratamiento quirúrgico. 8,13

En cuanto a las enfermedades que pueden suponer un mayor riesgo de sufrir STC, la diabetes presentó un riesgo ligeramente mayor, con un odd ratio de 1,179, frente a los pacientes que no la presentaban. La artritis reumatoide presentaba un riesgo superior frente a pacientes que no la padecían con resultados que no fueron estadísticamente significativos, de igual forma ocurrió con los pacientes que presentaron hiperuricemia, mayor riesgo pero no estadísticamente significativo (odd ratio 1,213$)(p=0,783)$, resultados en concordancia con otros trabajos publicados. ${ }^{8,12,13}$
Igualmente, se comprobó que la edad no presentaba un riesgo sobreañadido a la hora de padecer STC (odd ratio 0,933 ). De igual forma la obesidad presentaba un riesgo algo más elevado para derivar en el padecimiento de STC, (odd ratio 1,078 ) con respecto a las personas no obesas. La afectación de la mano no dominante tiene un riesgo menor que la mano dominante o la bilateral de sufrir STC (odd ratio 0,774 ), con resultado $p=0,388$, datos que muestran datos concordantes con lo existente en bibliografía previa. ${ }^{11,14}$

Se determinó un menor riesgo de presentar STC en el miembro no dominante (odd ratio 0,774 ), mientras que la edad de la población estudiada no supuso modificaciones en el riesgo de enfermedad (odd ratio), al igual que presenta el reciente trabajo de Harris-Adamson y col. ${ }^{15}$

Todos esos factores de riesgo que pueden desembocar en STC, se han considerado en multitud de estudios, alguno de los cuales son meta-análisis, lo que indica la validez externa de los resultados mostrados en el trabajo presentado; Spahn y col. ${ }^{16}$ demuestran que la prevalencia del STC experimenta un aumento según incrementa la edad; la mayor prevalencia se observó en pacientes de mediana edad (rango de 40 a 60 años). Otros factores significativos asociados en dicho trabajo al STC son, el sobrepeso o la obesidad (odd ratio $1,4(p<0,001)$ ). A su vez muestra que el STC se produce con mayor frecuencia en la mano dominante (odd ratio $1,8(p<0,001)$ ), en el género femenino (odd ratio $3,7(p<0,001))$ y en pacientes con diabetes mellitus (odd ratio 5,3 $(p<0,001)$ ); mientras que la publicación de Kozak y col., ${ }^{17}$ concluye en estudios primarios actuales sobre el riesgo de aparición del STC, que ese proceso patológico aumenta con trabajos de alta movilidad de la muñeca.

\section{Conclusión}

Se concluye, en base a los resultados mostrados, que existen determinadas profesiones que pueden aumentar el riesgo de padecer STC, así como otros factores adyacentes valorados como patologías endocrinas. Sin embargo, no se puede asumir como conclusión que exista una relación causal, es decir, que suponga una causa suficiente para desencadenarlo.

\section{Conflicto de Intereses}

Los autores declaran que no hay conflicto de intereses.

\section{Bibliografía}

1 Himmelstein JS, Feuerstein M, Stanek EJ III, et al. Work-related upperextremity disorders and work disability: clinical and psychosocial presentation. J Occup Environ Med 1995;37(11):1278-1286

2 Carragee EJ, Hentz VR. Repetitive trauma and nerve compression. Orthop Clin North Am 1988;19(01):157-164

3 Ohnari K, Uozumi T, Tsuji S. [Occupation and carpal tunnel syndrome]. Brain Nerve 2007;59(11):1247-1252

4 Latinovic R, Gulliford MC, Hughes RA. Incidence of common compressive neuropathies in primary care. J Neurol Neurosurg Psychiatry 2006;77(02):263-265

5 Papanicolaou GD, McCabe SJ, Firrell J. The prevalence and characteristics of nerve compression symptoms in the general population. J Hand Surg Am 2001;26(03):460-466 
6 Ettema AM, Amadio PC, Zhao C, et al. Changes in the functional structure of the tenosynovium in idiopathic carpal tunnel syndrome: a scanning electron microscope study. Plast Reconstr Surg 2006;118(06):1413-1422

7 Latko WA, Armstrong TJ, Franzblau A, Ulin SS, Werner RA, Albers JW. Cross-sectional study of the relationship between repetitive work and the prevalence of upper limb musculoskeletal disorders. Am J Ind Med 1999;36(02):248-259

8 Wieslander G, Norbäck D, Göthe CJ, Juhlin L. Carpal tunnel syndrome (CTS) and exposure to vibration, repetitive wrist movements, and heavy manual work: a case-referent study. $\mathrm{Br} \mathrm{J}$ Ind Med 1989;46(01):43-47

9 Bengtsson C, Hultén B, Larsson B, Noppa H, Steen B, Warnold I. [New weight and height tables for Swedish middle aged and elderly men and women]. Lakartidningen 1981;78(37):3152-3154

10 Iftikhar S, Javed MA, Kasuri MN. Frequency of Metabolic Syndrome and Its Components in Patients with Carpal Tunnel Syndrome. J Coll Physicians Surg Pak 2016;26(05):380-383

11 Day CS, Alexander M, Lal S, et al. Effects of workers' compensation on the diagnosis and surgical treatment of patients with hand and wrist disorders. J Bone Joint Surg Am 2010;92(13):2294-2299
12 van Rijn RM, Huisstede BM, Koes BW, Burdorf A. Associations between work-related factors and the carpal tunnel syndrome-a systematic review. Scand J Work Environ Health 2009;35(01): 19-36

13 Shiri R, Miranda H, Heliövaara M, Viikari-Juntura E. Physical work load factors and carpal tunnel syndrome: a population-based study. Occup Environ Med 2009;66(06):368-373

14 Gruson KI, Huang K, Wanich T, Depalma AA. Workers' compensation and outcomes of upper extremity surgery. J Am Acad Orthop Surg 2013;21(02):67-77

15 Harris-Adamson C, Eisen EA, Kapellusch J, et al. Biomechanical risk factors for carpal tunnel syndrome: a pooled study of 2474 workers. Occup Environ Med 2015;72(01):33-41

16 Spahn G, Wollny J, Hartmann B, Schiele R, Hofmann GO. [Metaanalysis for the evaluation of risk factors for carpal tunnel syndrome (CTS) Part I. General factors]. Z Orthop Unfall 2012;150(05):503-515

17 Kozak A, Schedlbauer G, Wirth T, Euler U, Westermann C, Nienhaus A. Association between work-related biomechanical risk factors and the occurrence of carpal tunnel syndrome: an overview of systematic reviews and a meta-analysis of current research. BMC Musculoskelet Disord 2015;16:231 